\title{
A Technique For Delineating Defects in Silicon.
}

\section{Luciano Mule'Stagno}

\author{
MEMC Electronic Materials Inc., Silicon Materials Research Group, Crystal Technology Dept. \\ 501 Pearl Dr., St. Peters, MO 63376, USA
}

Keywords: interstitials, interstitial defects, etching, copper.

\begin{abstract}
A decoration and etching technique was developed to delineate several types of defects in silicon wafers, slugs and slabs. The technique was originally developed to detect interstitial type (A) defects but it has proved highly effective in decorating all kinds of other defects. Being fast, and requiring no special equipment except an inexpensive muffle furnace and a dedicated etch bench the technique has quickly become an integral part of our characterization portfolio. We discuss below how we have used this technique and its advantages over other methods used to detect Adefects.
\end{abstract}

\section{Introduction}

Interstitial-type extended defects in Silicon occur when a Silicon Crystal is grown under conditions that result in excess silicon interstitials being incorporated in the lattice. The incorporation of point defects into the cooling crystal occurs very close to the melt-solid interface. Intrinsic point defects occur in equilibrium at the crystallization temperature. Which species survives (vacancies or interstitials) is determined by the ratio of the pulling speed to the axial temperature gradient near the growth interface [1,2]. The critical V $\backslash \mathrm{G}$ ratio is about $0.2 \mathrm{~mm} / \mathrm{minK}^{2}$. Crystals grown with a higher ratio would be vacancy rich, lower ratio, interstitial rich. As the crystal cools these excess vacancies agglomerate into voids (COPs) $100-200 \mathrm{~nm}$ in size, excess interstitials form dislocation tangles 1-2 $\mu \mathrm{m}$ in length [3,4]. Unlike COPs which cause GOI failure, it is believed that interstitial-type defects result in diffusion pipes or spikes in the p-n junction. Though there is still debate as to the mechanism for device failure caused by these defects, it is generally agreed that their presence is undesirable. Some studies suggest that they are harmful even if undecorated. If in addition they become decorated with metals, for which they are an excellent gettering site, their negative effect is amplified [5].

Typically, interstitial or A-defects occur in densities of $10^{4} / \mathrm{cm}^{3}$ or less which makes them difficult to find. In addition, they are undetected in many of the common tests used today such as OISF testing, GOI testing and Cleave and Etch. Traditional methods for detecting A-defects have included a Secco [6], Yang [7] or similar etch which shows them up as etch pits. Though these methods can be somewhat quantitative and work on polished wafers, the result can be difficult to interpret on rough-cut slugs or slabs due to pits caused by damage and contamination. In addition it requires extensive microscopic evaluation at medium to high magnification which makes it time consuming. We report here a technique that detects interstitial-type defects and provides qualitative information that can be obtained with the naked eye (or under bright light) and is macroscopic in nature. For this reason the technique has proven invaluable for tuning new crystal processes under development. In addition we have expanded the use of the technique to the macro-detection of many other types of defects in silicon. Since almost any defect in the lattice will getter copper, the technique can be used to delineate most common defect types.

\section{Experimental}

A high concentration of Copper Nitrate $\left(\mathrm{Cu}\left(\mathrm{NO}_{3}\right)_{4} \cdot 5 \mathrm{H}_{2} 0\right)$ solution is prepared and spread in a thin film on the back of the sample (Figure 1). The spreading is done by smearing or spraying on the back of the sample which can be a wafer, slug or slab. The sample is then heated at $50-60^{\circ} \mathrm{C}$ on a hot-plate to dry the solution (Figure 2). Once the solution has dried, which results in a dry layer of Copper Nitrate on the back of the sample, the sample is annealed for 10-20 minutes at $900^{\circ} \mathrm{C}$ in a muffle furnace and air-quenched to room temperature (Figures 3 and 4). The anneal is longer for thicker samples (shougs and slabs), shorter for thinner samples (wafers). The sample is then etched to a mirror finish using a mixed acid etchant mixture (57\% Nitric Acid (70\%), 18\% Hydrofluoric Acid (49\%) and 25\% Hydrochloric Acid), followed by Secco Etch (0.15M Potassium Dichromate and 49\% Hydrofluoric Acid, 1:2 Ratio). After rinsing and drying the sample is ready for inspection under bright or room light (Figure 5). 


\section{Results}

The main motivation for the development of this technique was the need for a reliable quick way to detect interstitial type defects. Prior to this technique we detected interstitial defects in wafers as etch pits after Secco or other decorative etches (Figure 6). Though this technique works fairly well on wafers and can be used to get quantitative information about defect densities, it is less reliable on unpolished surfaces (such as slabs or slugs) due to etching artifacts. In addition it is very time consuming, especially when a 2-dimensional picture is desired (Figure 7). Occasionally the etch pits are large enough to be visible with the naked eye under bright light, but this is not always the case. The etch pits can be seen in most cases by a laser scattering tool on an etched wafer. The disadvantage of this technique is that it works only on wafers and requires a dedicated and expensive light scattering tool.

On the other hand, the technique described here is fast, requires no special dedicated equipment (that is usually not already present), and provides 2-Dimensional information on defect distribution. Figure 5 shows images of $200 \mathrm{~mm}$ wafer quarters decorated with this technique. Both wafers have a vacancy-interstitial boundary within the crystal, but in the case of the first wafer there is also an A-defect band, which is very easy to detect. It is also evident that D-defects are also decorated and made easily apparent. This technique is also very effective at decorating different defect regions within the vacancy regime as can be seen by examining the edge of the vacancy region in Fig. 5.

Figure 8 shows a research crystal which has been slabbed longitudinally. The pull-rate in this crystal was oscillated to move from vacancy to interstitial and back to a vacancy region. The void and interstitial regions are easily seen separated by a thick OISF Band. On closer examination of the transition region, one can also distinguish a precipitation band region outside the OISF band and a narrow defect free region on the interstitial side. The higher precipitation region outside the OISF band is created because coolong conditions favored the clustering of vacancies with oxygen rather than void formation. The defect-free region is a region where the concentration of interstitials is too low to result in interstitial defect agglomerates [1,2]. These images also hilight the fact that this technique makes it very easy to distinguish defect-free regions from areas containing voids or interstitial defects. Figure 9 shows a tail and taper longitudinal slab. In the tail one can clearly distinguish an area of heavier precipitation formed due to quenching of oxygen clusters when the tail was popped out and moved away from the melt. The dislocations in the seed attached to the taper are also very easily observed. Figure 10 shows two more examples of wafers decorated with this technique. The wafer on the left is fully vacancy rich with a higher precipitation band at the edge but no OISF ring. The one on the right shows an OISF ring and an outer L-Band region [2].

Though the technique is being used routinely to obtain qualitative defect information, it is also possible to use these samples to get an idea of the defect density. Figure 11 shows one such example. The region that was measured had Adefects that started at a very high density and quickly decresed to a very low density. To calculate the volume density, the defect area density is measured by means of a microscope and converted to a volume density using the etching removal of the decorative etch. If the defect size after etching is significant compared to the etching removal, its dimensions must be added to the etching removal to obtain an accurate volume density. From the graph in Figure 11 it can bee seen that even very low defect densities in the $10^{3}-10^{4}$ defect $/ \mathrm{cm}^{3}$ are revealed in these samples. Few other techniques can reveal the presence of defects present at such low densities.

\section{Summary}

A new technique is described that makes use of Copper decoration in conjunction with non-defect delineating and defect delineating etches. The main purpose of the technique was to detect the presence of interstitial-type defects, but it has been found to delineate most lattice defects. Though it does not easily provide quantitative data about the defects, it provides excellent qualitative data on any type of silicon sample.

\section{Acknowledgements}

Bob Falster for his contribution in developing this technique. Blake Rowe, Derrick Smith, Steve Mecker and Jim Cole for providing some of the images used in this paper and their help in further perfecting the technique. Milind Kulkarni, Joe Holzer, Seamus McQuaid, Steve Markgraf, Jeff Libbert, Rich Phillips and Ann Grabau for helpful discussions and their role in the initial use of the technique. 


\section{References}

[1] V. V. Voronkov, J. Crystal growth, 59, 625 (1982)

[2] R. Falster, V. V. Voronkov, J. Holzer, S. Markgraf, S. McQuaid and L. Mule'Stagno, Semiconductor Silicon 1998, H.R. Huff, U, Gosele, H. Tsuya editors, 468-489, The Electrochemical Soc., Pennington, NJ (1998).

[3] J. Ryuta, E. Morita, T. Tanaka, Y. Shimanuki, Jap. Journal of Appl. Phys., 29, 11, L1947-L1949 (1990)

[4] H. Foell, and B. O. Kolbesen, Appl Phys. 8, 319(1975).

[5] G. H. Plantiga, IEEE Trans Electron Devices ED-16, 394-400 (1969).

[6] F. Secco d'Aragona, J. Electrochem. Soc., 119, 948 (1972).

[7] K. H. Yang, J. Electrochem. Soc., 131, 1140 (1984).

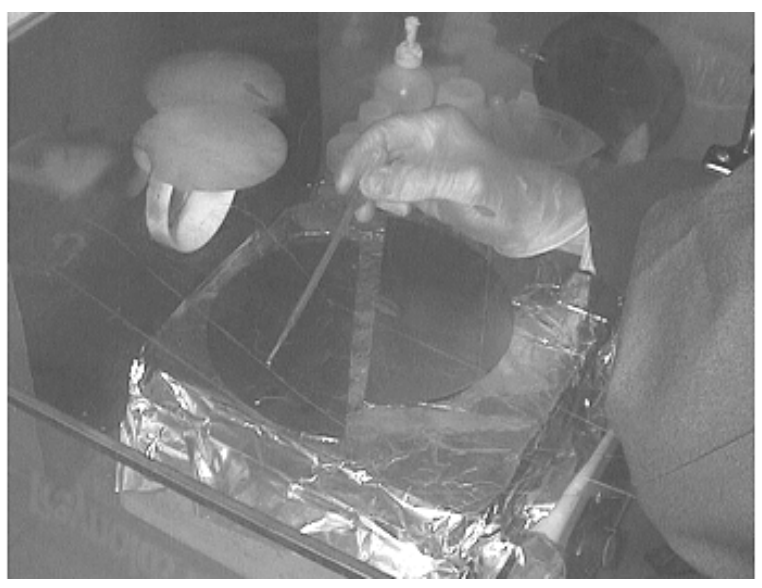

Figure 1 - Copper Nitrate application.

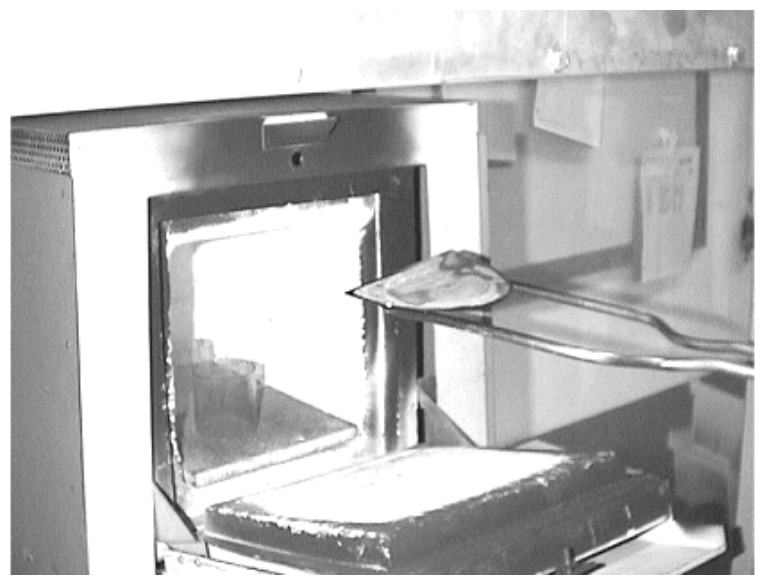

Figure 3 - Sample (1/2 wafer) being inserted into hot furnace.

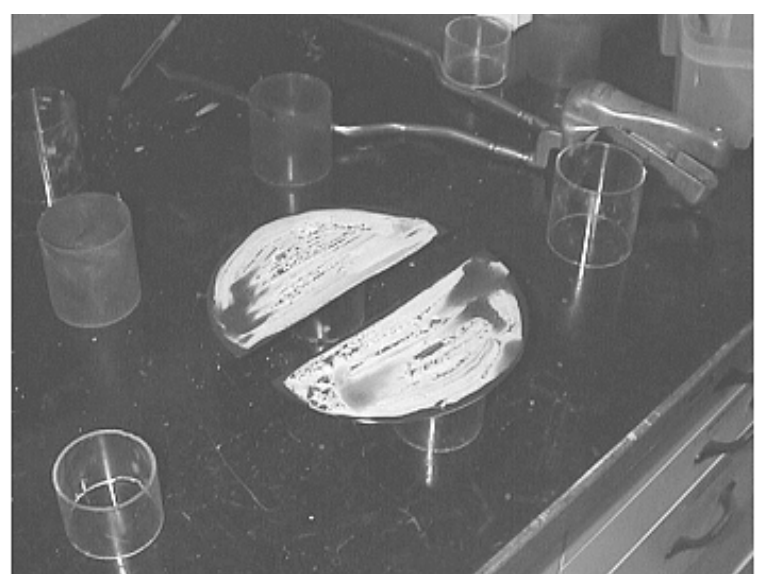

Figure 2 - Drying of Copper Nitrate on the back of the sample.

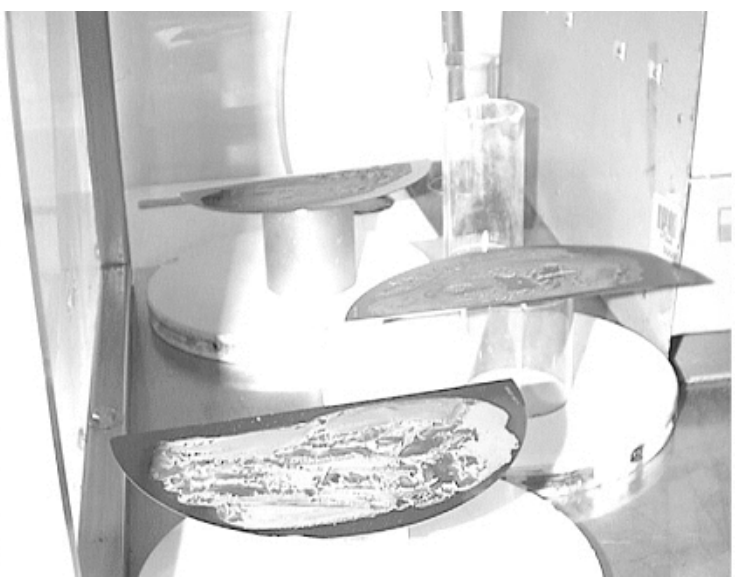

Figure 4 - Samples after air-quenching ready for cleaning and etching. The Copper Nitrate color $\mathrm{c}$ changes from blue or green to white 

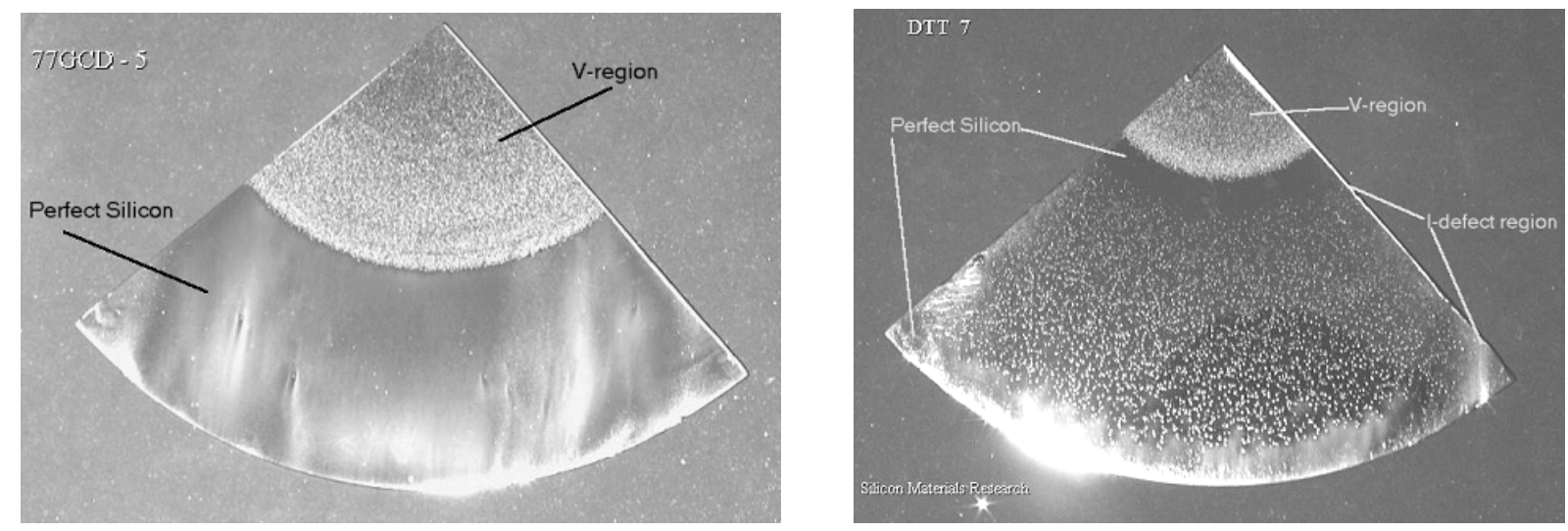

Figure 5 - Two quarter wafer samples decorated with the technique. The sample on the left has a vacancy core surrounded by a defect-free region. The sample on the right has interstitial-type (A) defects in the outer region.

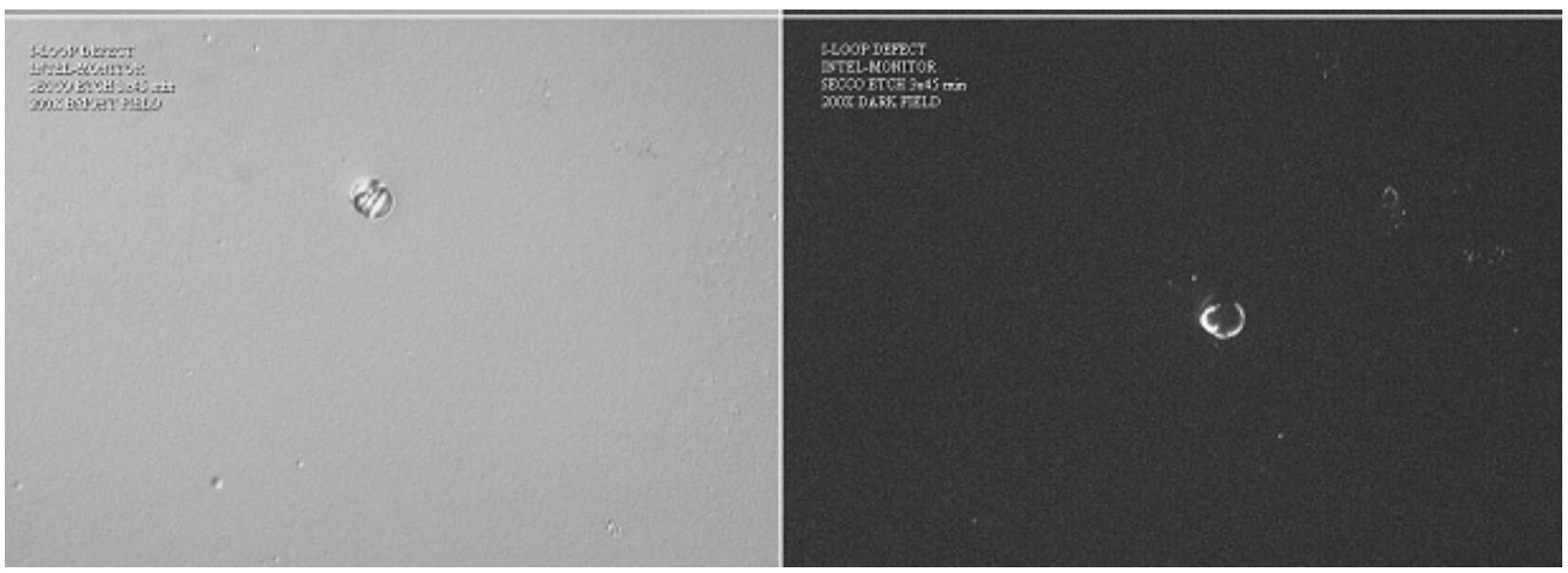

Figure 6. Bright (left) and darkfield images of etch pits caused by interstitial defects on a silicon wafer. Images were taken at $400 \mathrm{X}$ 

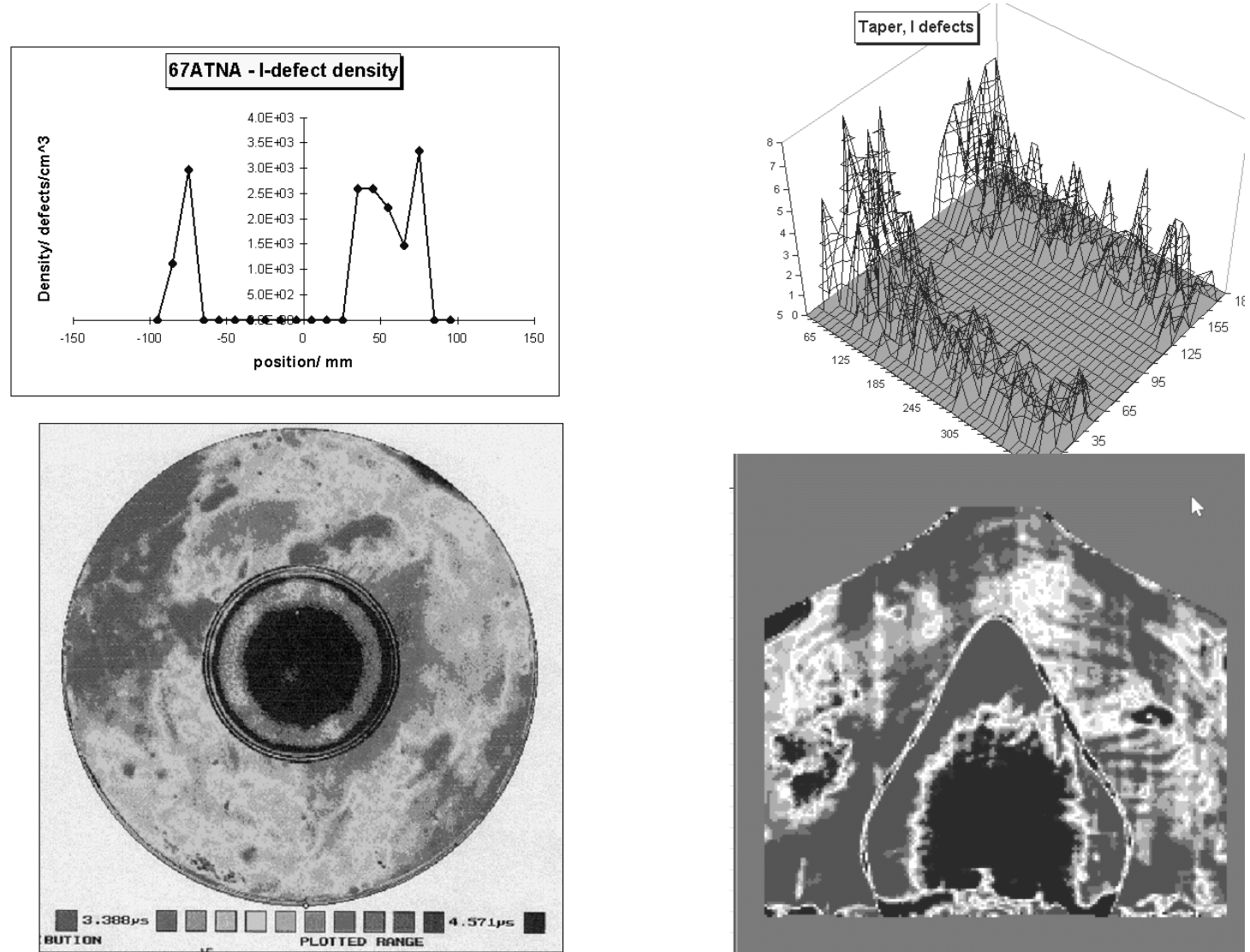

Figure 7. Interstitial defect density (top) obtained by counting defects at 400X across several diameters of a wafer (left) and slab of a top section of a crystal (right, including taper). Though the defects are identified in the right locations, it is difficult and very time consuming to obtain a good overall picture using this technique. The lower images shows a lifetime map of the wafer and slab after heat treatment. The interstitial defect region is not hi-lighted by heat-treating and Recombination Lifetime. The OISF ring and vacancy regions are clearly seen in the lifetime scans.

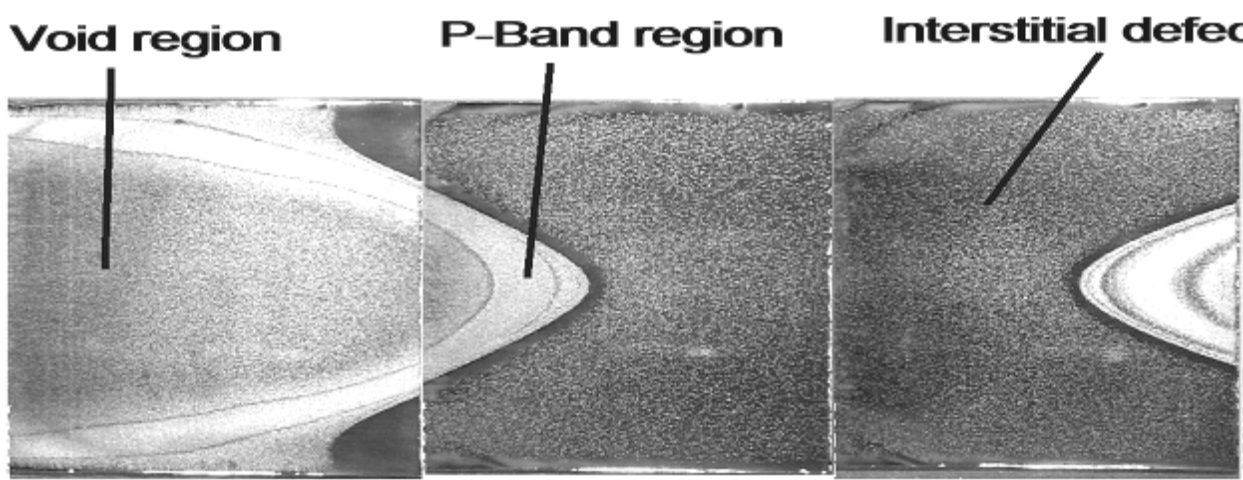

Seed-end

Opposite-end

Figure 8 - Image of a slabbed research crystal. In this crystal the pull-rate was oscillated to transition from a vacancy, to an interstitial and back to a vacancy region. 

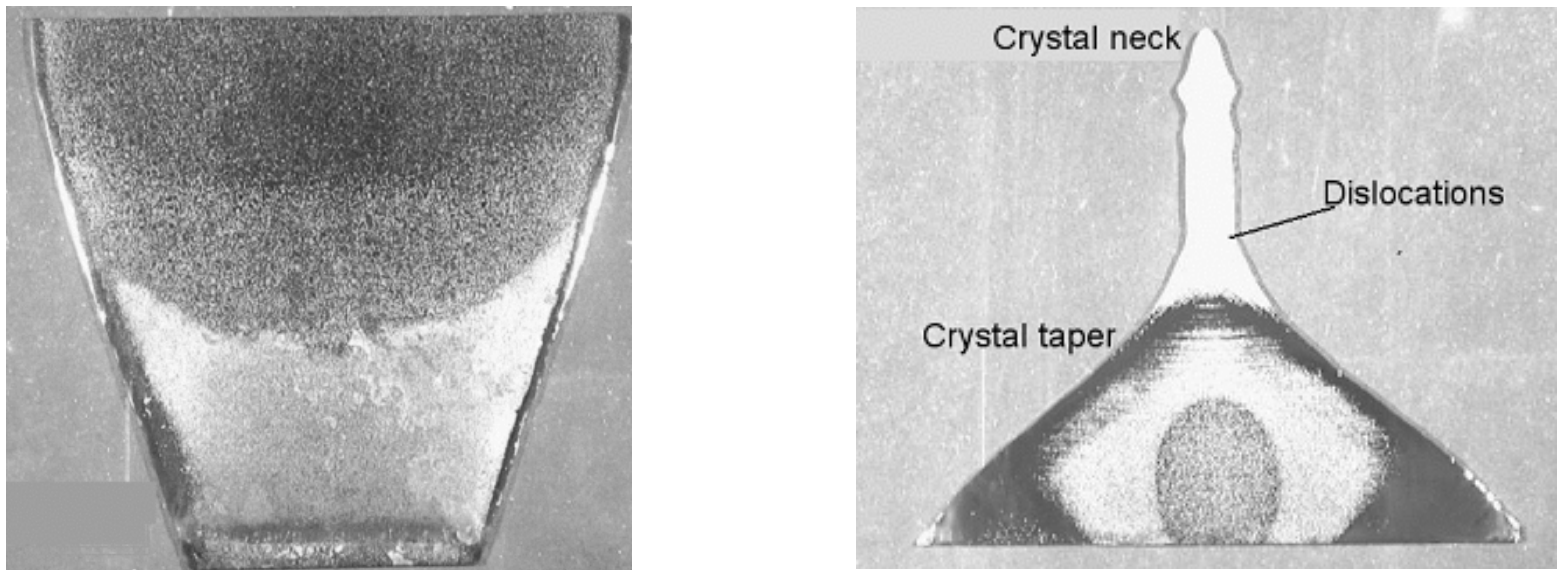

Figure 9 - Images of slabbed tail (upper part) and taper with neck. A quenching front (region of higher precipitation) is clearly visible in the tail, as are the dislocations in the neck.
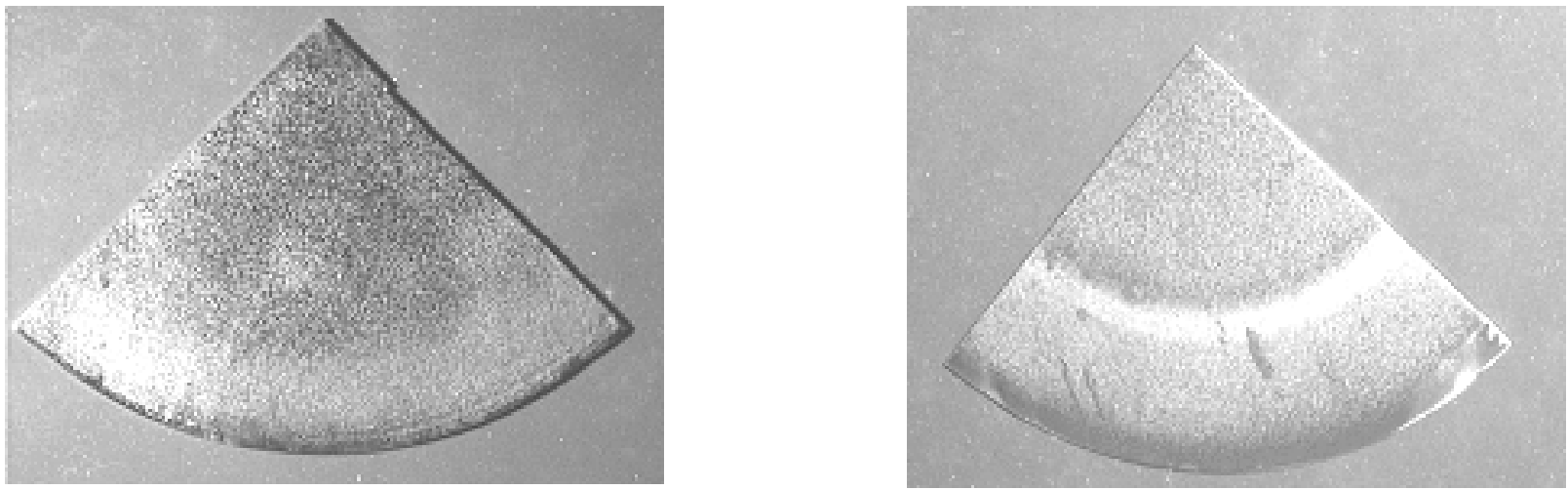

Figure 10 - Images of wafer quarters. The one of the left is fully vacancy. The higher density region close to the edge corresponds to the $\mathrm{H}$ band [2]. The sample on the right has an OISF band about halfway to the edge and an extended Lband beyond this.
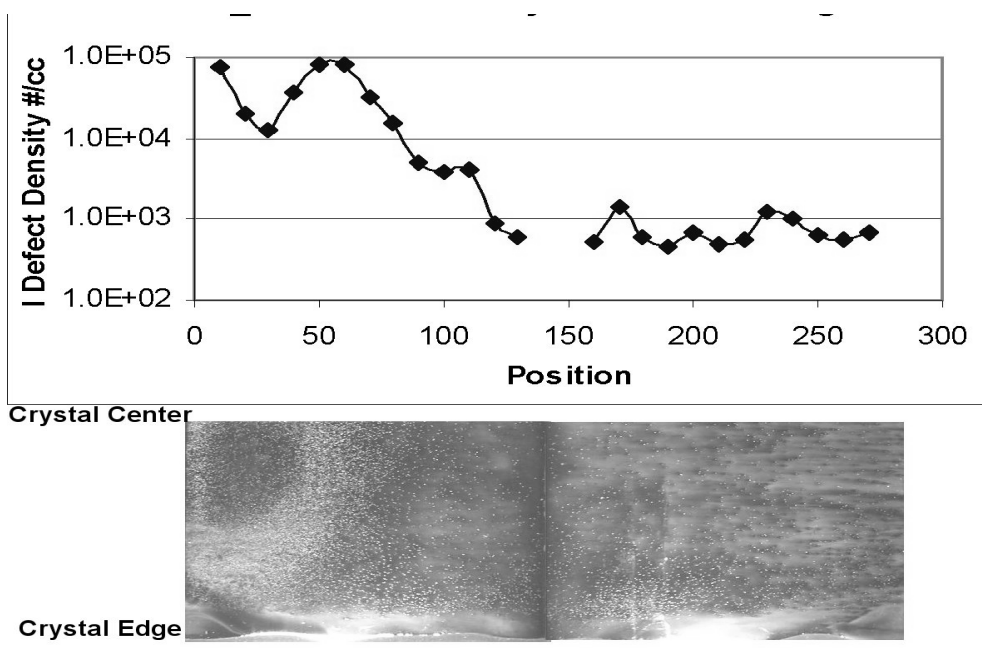

Figure 11 - Plot of the interstitial defect density in a research crystal. This part of the crystal was grown interstitial rich, but with an interstitial defect density that started very high and quickly dropped to a lower density. 